\title{
Efficacy and Safety of Janus Kinase Inhibitors for the Treatment of Atopic Dermatitis: A Systematic Review and Meta-Analysis
}

\author{
Chenyang $\mathrm{Li}^{\mathrm{a}}$ Xun Sun ${ }^{\mathrm{b}}$ Kun Zhao ${ }^{c}$ Fanxiang Meng ${ }^{\mathrm{b}} \mathrm{Lin}^{\mathrm{L}}{ }^{\mathrm{a}}$ \\ Zhenzhen $\mathrm{Mu}^{\mathrm{a}}$ Xiuping $\mathrm{Han}^{\mathrm{a}}$ \\ ${ }^{a}$ Department of Dermatology, Shengjing Hospital of China Medical University, Shenyang, China; ${ }^{\mathrm{b}}$ Department \\ of Immunology, China Medical University, Shenyang, China; 'Department of Sport Medicine, Zhejiang University \\ School of Medicine, Hangzhou, China
}

\section{Keywords}

Janus kinase inhibitors · Atopic dermatitis · Meta-analysis

\begin{abstract}
Background: Current therapeutic options for atopic dermatitis (AD) are limited. Janus kinase (JAK) inhibitors may be viable alternatives. Objectives: To assess the efficacy and safety of JAK inhibitors for AD treatment. Methods: We searched PubMed, Embase, the Cochrane Controlled Register of Trials, Web of Science, Global Resource of Eczema Trials database, and ClinicalTrials.gov from inception to September 1, 2020. Randomized clinical trials (RCTs) comparing JAK inhibitors with placebo/vehicle treatment for AD patients were included. The primary study outcomes included (1) the change (\%) from the Eczema Area and Severity Index (EASI) baseline expressed as weighted mean difference (WMD) and 95\% confidence interval $(95 \% \mathrm{Cl})$, and (2) the Investigator's Global Assessment (IGA) response and safety outcomes expressed as relative risk (RR) and $95 \% \mathrm{Cl}$. Results: We included $14 \mathrm{RCTs}$ published in 13 studies (3,822 patients). Treatment with JAK inhibitors significantly improved IGA response (RR 2.83, 95\% $\mathrm{Cl} 2.25-3.56, p<0.001$ ) and EASI score (WMD $-28.82,95 \% \mathrm{CI}$ -34.48 to $-23.16, p<0.001)$. JAK inhibitor treatment achieved
\end{abstract}

karger@karger.com www.karger.com/drm

Karger $\frac{1}{\%}$
(C) 2021 The Author(s)

Published by S. Karger AG, Basel

This is an Open Access article licensed under the Creative Commons Attribution-NonCommercial-4.0 International License (CC BY-NC) (http://www.karger.com/Services/OpenAccessLicense), applicable to the online version of the article only. Usage and distribution for commercial purposes requires written permission. the largest improvement in both IGA response (RR 3.59, 95\% $\mathrm{Cl} 2.66-4.84, p<0.001)$ and EASI score (WMD $-42.00,95 \% \mathrm{Cl}$ -48.64 to $-35.36, p<0.001$ ) by week 4 of treatment. Topical JAK inhibitors were significantly more efficacious than oral inhibitors. Upadacitinib treatment for 4 weeks was most effective in reducing EASI score (WMD $-53.92,95 \% \mathrm{Cl}-69.26$ to $-38.58, p<0.001$ ), while abrocitinib for 4 weeks led to the most effective IGA response (RR 5.47, 95\% CI 2.74-10.93, $p<$ 0.001 ). There was no difference in the frequency of adverse events (AEs) leading to discontinuation; however, JAK inhibitors use, especially abrocitinib, led to a higher incidence of treatment-emergent AEs (RR 1.25, 95\% Cl 1.10-1.42, $p=$ 0.001). Conclusion: Our results imply that JAK inhibitors are an effective and safe AD treatment. Nevertheless, further trials with longer duration and head-to-head comparisons of different JAK inhibitors are needed.

C 2021 The Author(s).

Published by S. Karger AG, Basel

\section{Introduction}

Atopic dermatitis $(\mathrm{AD})$ is a chronic inflammatory skin disease that is characterized by eczematous lesions and pruritus [1]. It is one of the most common skin disorders
Correspondence to:

Xiuping Han, licy1470721@163.com 
worldwide, affecting approximately $20 \%$ of children and $2.1-4.9 \%$ of adults $[2,3]$. AD etiologies include environmental factors, genetic disposition, immune dysregulation, and impaired skin barrier function [1]. AD imposes psychological and social burdens, associated with a higher risk of depression, anxiety, work absenteeism, and suicidality [4-7].

Topical anti-inflammatory agents and corticosteroids constitute primary treatment for $\mathrm{AD}$. However, the prognosis is typically poor, mainly due to the low efficacy of the drugs used and marked adverse effects. Specifically, topical anti-inflammatory agents are ineffective in moderate-to-severe $\mathrm{AD}$, and corticosteroids can have serious side effects $[8,9]$. Biological therapies have advanced AD treatment by reducing the expression of pathogenic inflammatory factors [10]; however, such therapies are immunogenic and may lead to loss of efficacy. Some patients remain unresponsive $[11,12]$.

$\mathrm{AD}$ is characterized by multi-cytokine polarization, and a therapeutic approach capable of inhibiting more than one cytokine cascade from increasing efficacy is theoretically appealing $[1,13]$. Cytokines that can contribute to the pathophysiology of AD include IL-4, IFN- $\gamma$, IL-31, IL-13, IL-23, and IL-22, as they interact with both type I and II cytokine receptors. The Janus kinase (JAK) family, comprising JAK1, JAK2, JAK3, and tyrosine kinase 2, are cytoplasmic tyrosine kinases. Both type I and II cytokine receptors lack intrinsic enzyme activity and depend on the JAKs for signal transduction [14]. Specifically, JAKs bind to intracellular chains of the cytokine receptor to generate functional signaling complexes and regulate the inflammatory process by activating intracytoplasmic transcription factors, i.e. the signal transducer and activator of transcription (STAT) molecules. Upon activation, STAT proteins dimerize, translocate into the nucleus, and either positively or negatively regulate downstream target gene expression, namely, inflammatory mediators $[14,15]$. Thus, inhibiting JAK activity may be more effective than targeting a single cytokine. Further, JAK repression due to disruption of cytokine signaling attenuates a chronic itch propelled by type- 2 cytokine and receptor interplay in sensory neurons [16]. JAK repression also improves skin barrier function by regulating the expression of filaggrin, a skin barrier protein [17]. These observations imply that JAK is an adaptable target for $\mathrm{AD}$ treatment, along with its neuronal mechanisms of action and its role in regulating epithelial barrier function and immune response.

There have been researches on the evidence of JAK inhibitors for the treatment of other immune-mediated skin disorder, including psoriasis and alopecia areata [18, 19]. Recently, several studies have also evaluated the use of various administration routes and multiple JAK inhibitors among different populations to treat AD. Here, this study evaluates available evidence on the efficacy and safety outcomes for treatment duration, administration route, and types of JAK inhibitors for treating $\mathrm{AD}$.

\section{Materials and Methods}

This systematic review and meta-analysis was conducted in accordance with the Preferred Reporting Items for Systematic Reviews and Meta-analyses (PRISMA) guidelines (online supplementary Appendix 1) $[20,21]$. It is registered in PROSPERO (http://www.crd.york.ac.uk/prospero) with registration number CRD42020215945.

\section{Search Strategy}

We searched electronic databases (PubMed, Embase, the Cochrane Controlled Register of Trials, Web of Science, the Global Resource of Eczema Trials database, and ClinicalTrials.gov) without language restriction, from the inception to September 1, 2020. Full articles, systematic and literature reviews were retrieved and searched to identify additional eligible studies. The detailed search strategies were independently evaluated by a third investigator and are provided in online supplementary Table 1.

\section{Study Selection}

We included randomized clinical trials (RCTs) on AD treatment that compared JAK inhibitors with placebo/vehicle. We excluded duplicate publications, nonhuman studies, conference abstracts, and studies wherein data for outcomes of interest were not available. The eligibility of all articles was assessed by two investigators, and differences of opinion were settled by consensus or after consultation with a third investigator.

Data Extraction and Quality Assessment

Two reviewers independently extracted and cross-checked the data to avoid errors. Information extracted from the selected studies include:

- Country

- ClinicalTrials.gov Identifier

- Diagnosis criteria

- AD severity

- Baseline characteristics of patients, including sample size, age and gender percentage

- Treatment, including type and dosage of control and JAK inhibitors

- Outcomes of interest

- Timepoint of outcomes

Efficacy outcomes evaluated were (1) Investigator's Global Assessment (IGA) response (clear [0] or almost clear [1] with an improvement of $\geq 2$ grades from baseline); and (2) percent change from baseline in the Eczema Area and Severity Index (EASI). Safety outcomes were adverse events (AEs) leading to discontinuation and treatment-emergent AEs (TEAEs). When outcomes were presented in figures, WebPlotDigitizer (version 4.3, https://apps.au- 


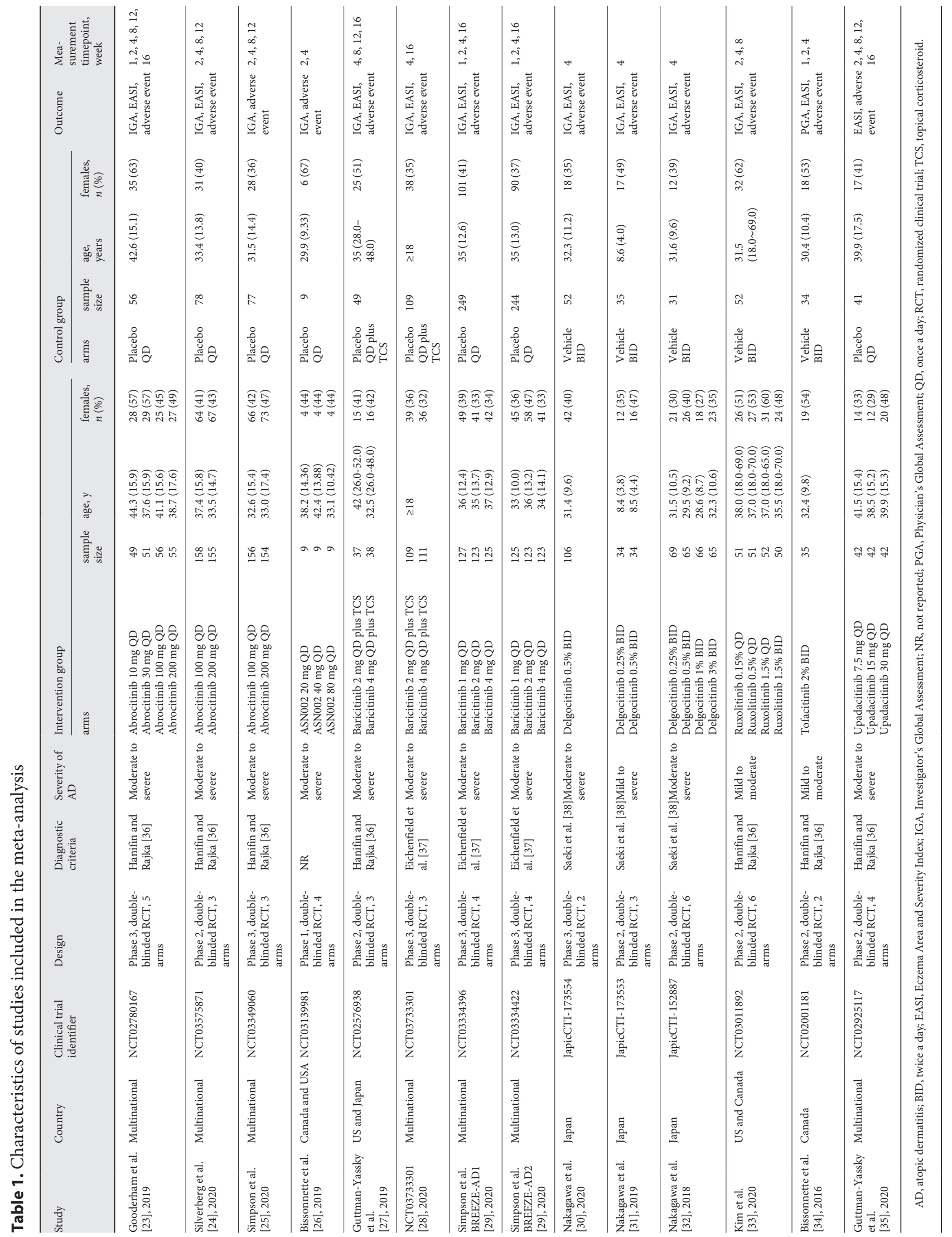


tomeris.io/wpd/index.zh_CN.html) was used to extract data from the figure. In studies without a placebo or vehicle comparator arm, where patients subsequently entered an open-label period, we extracted data from the placebo/vehicle-controlled period. When there was an active control arm, we only extracted data from the placebo or vehicle-control arm.

We evaluated the quality of included articles and the risk of bias using the Cochrane Collaboration's Risk of Bias assessment tool [22]. Quality parameters assessed were (1) random sequence generation; (2) allocation concealment; (3) blinding of participant and personnel; (4) incomplete outcome data; (5) selective reporting; and (6) other bias.

\section{Statistical Analyses}

Statistical analyses were performed using Review Manager, version 5.4 (The Cochrane Collaboration) and Stata version 12.0 (StataCorp LP). We calculated relative risk (RR) with 95\% CIs for dichotomous outcomes and weighted mean difference (WMD) with 95\% CIs for continuous outcomes. $p<0.05$ (2-tailed) was considered statistically significant. $I^{2}$ was used to assess heterogeneity $\left(I^{2} \geq 50 \%\right.$ considered significant). If significant heterogeneity was present, the data were assessed using a random-effects model; otherwise, a fixed-effects model was utilized. We conducted subgroup analyses based on treatment duration, administration route, baseline severities of AD and types of JAK inhibitors. Funnel plots and Egger tests assessed publication bias.

\section{Results}

\section{Search Results and Study Characteristics}

The database search identified 878 relevant records. After removing 221 duplicates, an additional 625 records were excluded based on title and abstract review. Nineteen records were excluded on full-text assessment. Fourteen RCTs published in 13 studies [23-35] were included in the systematic review and meta-analysis (online supplementary Fig. 1).

The study included 3,822 patients. Both topical and oral administration of JAK inhibitors was assessed. All studies used placebo or vehicle ointment as controls. The vehicle used was the base cream or ointment without active ingredient. The detailed characteristics of the selected 13 studies are summarized in Table 1 [23-35].

\section{Quality Assessment}

While 12 studies specified methods of random sequence generation [23-28, 30-35], one study had an "unclear" bias [29]. Further, 9 studies had described allocation concealment [23-25, 27-29, 31, 34, 35], and 4 studies had an "unclear" bias [26, 30, 32, 33]. Next, 8 studies had blinded outcome assessment [23-26, 28, 33-35], and 11 studies had reported a "low" risk of incomplete outcome data [24-26, 28-35]. All 13 studies had a "low" risk of performance bias and reporting bias, but an "unclear" risk of other biases [23-35] (online supplementary Fig. 2).

\section{Efficacy Outcomes}

Overall Clinical Efficacy

As measurement time points were different in each of the 13 studies, we pooled data for the last time point of each study during primary analysis.

\section{IGA Response of Patients}

A meta-analysis of 12 studies including 3,537 patients using a fixed-effects model showed a significantly improved IGA response among patients treated with JAK inhibitors compared to those treated with placebo/vehicle (RR 2.83, 95\% CI 2.25-3.56, $p<0.001$ ). No heterogeneity was detected $\left(I^{2}=0 \%\right)$ (Fig. 1$)$.

\section{Percent Change from Baseline in EASI}

A meta-analysis of 11 studies including 3,242 patients using a random-effects model showed a significant decrease in EASI score in JAK inhibitors group compared with the placebo/vehicle group (WMD -28.82, 95\% CI -34.48 to $-23.16, p<0.001)$. However, significant heterogeneity was detected among studies $\left(I^{2}=50 \%\right)$ (Fig. 2).

\section{Clinical Efficacy by Treatment Duration}

A subgroup analysis was performed by pooling studies with the same treatment durations. A significant improvement in IGA response was found in the JAK inhibitor treatment group compared to placebo/vehicle at each time point with no heterogeneity (Table 2 ). The largest improvement occurred at week 4 in the JAK inhibitor treatment groups compared with the placebo/vehicle group (RR 3.59, 95\% CI 2.66-4.84, $p<0.001$ ) (Table 2).

Similar efficacy was reported in EASI. JAK inhibitors showed a significant decrease in the EASI score at all time points compared to placebo/vehicle (Table 3). The largest reduction of EASI score occurred at week 4 in the JAK inhibitors treatment group compared to the placebo/vehicle group (WMD $-42.00,95 \%$ CI -48.64 to $-35.36, p<$ 0.001) (Table 3).

\section{Clinical Efficacy by Administration Route}

A subgroup analysis was performed by pooling studies using 1 of 2 available administration routes (oral and topical) with the same treatment durations. Both oral and topical administration of JAK inhibitors was associated with significantly improved IGA response compared with placebo/vehicle at each treatment time point, with no heterogeneity. The largest improvement in IGA re- 


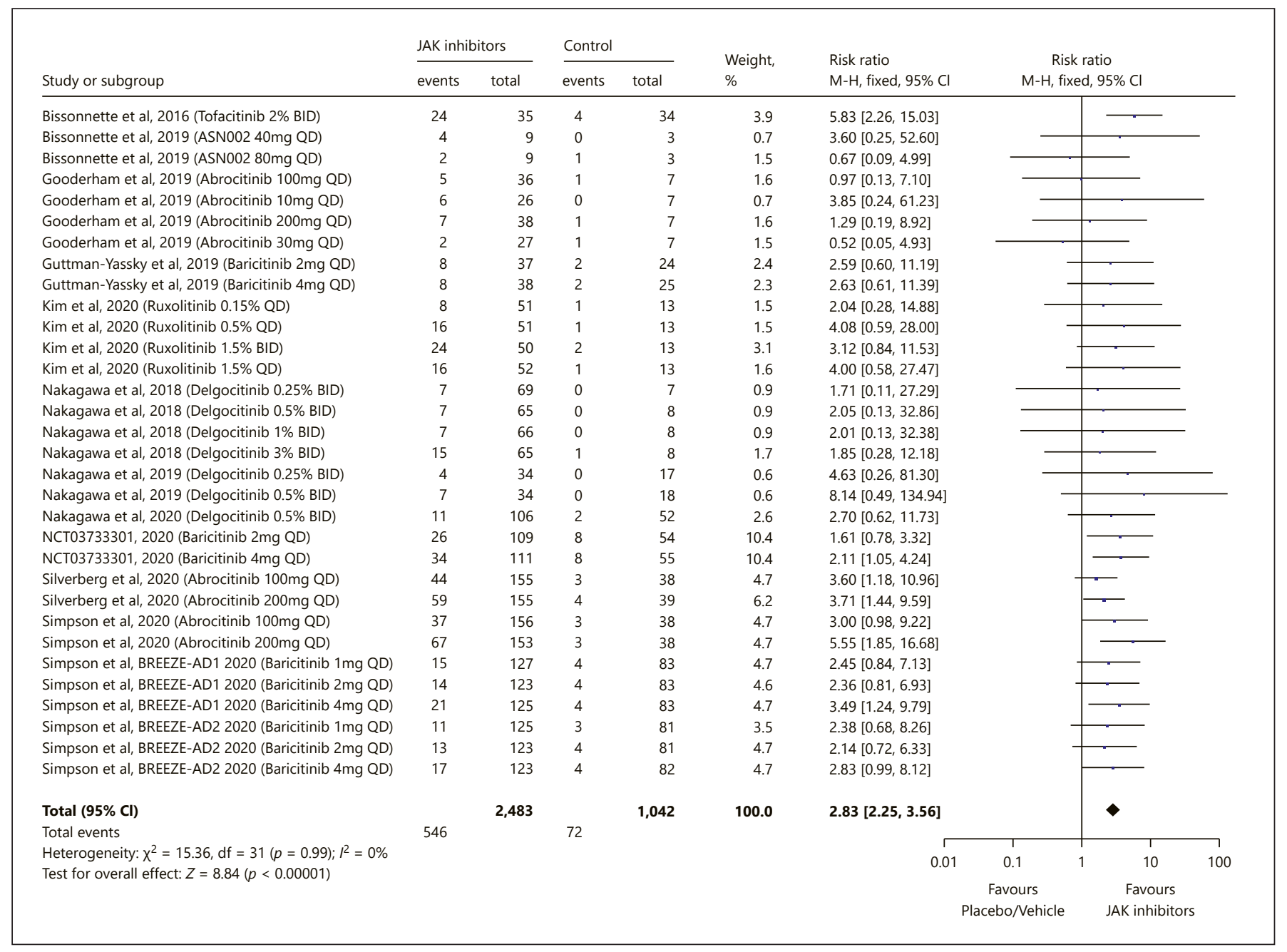

Fig. 1. Meta-analysis for overall efficacy of treatment with Janus kinase (JAK) inhibitors in patients achieving Investigator's Global Assessment response versus placebo/vehicle.

sponse by oral administration occurred at week 4 (RR $3.65,95 \%$ CI $2.53-5.27, p<0.001$ ), and the largest improvement by topical administration occurred at week 2 (RR 3.53, 95\% CI 1.43-8.73, $p=0.006$ ) (Table 2).

Both oral and topical administration of JAK inhibitors significantly reduced the EASI score compared with placebo/vehicle at each treatment time point. The largest reduction in EASI of both oral (WMD -37.66, 95\% CI -49.06 to $-26.27, p<0.001)$ and topical administration (WMD $-46.82,95 \%$ CI -54.56 to $-39.08, p<0.001$ ) occurred at week 4 (Table 3 ).

\section{Clinical Efficacy by Baseline Severity}

For a pooled subgroup analysis of studies with the same baseline severity of AD classified as mild-to-severe, mild-to-moderate, or moderate-to-severe, efficacy at week 4 was the time point for analysis due to insufficient data for other treatment durations. For EASI, topical administration demonstrated a greater effect compared to oral administration in the moderate-to-severe subgroup (topical: WMD $-44.16,95 \%$ CI -54.69 to $-33.63, p<$ 0.001 , oral: WMD $-37.66,95 \%$ CI -49.06 to $-26.27, p<$ 0.001 ) (online supplementary Table 2).

\section{Clinical Efficacy by Type of JAK Inhibitors}

For a pooled subgroup analysis of studies using the same JAK inhibitors for the same treatment duration, efficacy at week 4 was the time point for this analysis based on prior results. We assessed six JAK inhibitors (abrocitinib, ASN002, baricitinib, delgocitinib, rux- 


\begin{tabular}{|c|c|c|c|c|c|c|c|c|c|c|c|}
\hline \multirow{3}{*}{$\begin{array}{l}\text { Study or subgroup } \\
\text { Bissonnette et al, } 2016 \text { (Tofacitinib 2\% BID) }\end{array}$} & \multicolumn{3}{|c|}{ JAK inhibitors } & \multicolumn{3}{|l|}{ Control } & \multirow{3}{*}{$\begin{array}{l}\begin{array}{l}\text { Weight, } \\
\%\end{array} \\
4.3\end{array}$} & \multirow{3}{*}{$\begin{array}{l}\text { Mean difference } \\
\text { IV, random, 95\% Cl } \\
-51.80[-69.50,-34.10]\end{array}$} & \multirow{2}{*}{\multicolumn{3}{|c|}{$\begin{array}{l}\text { Mean difference } \\
\text { IV, random, } 95 \% \mathrm{Cl}\end{array}$}} \\
\hline & \multirow{2}{*}{$\begin{array}{r}\text { mean } \\
-81.7\end{array}$} & \multirow{2}{*}{$\frac{S D}{36.79}$} & \multirow{2}{*}{ total } & \multirow{2}{*}{$\begin{array}{r}\text { mean } \\
-29.9\end{array}$} & \multirow{2}{*}{$\frac{S D}{35.97}$} & \multirow{2}{*}{ total } & & & & & \\
\hline & & & & & & & & & & & \\
\hline Gooderham et al, 2019 (Abrocitinib 100mg QD) & -27.66 & 54.57 & 36 & -35.91 & 47.71 & 7 & 1.6 & $8.25[-31.33,47.83]$ & & & \\
\hline Gooderham et al, 2019 (Abrocitinib 10mg QD) & -45.24 & 44.58 & 26 & -35.91 & 47.71 & 7 & 1.6 & $-9.33[-48.61,29.95]$ & & & \\
\hline Gooderham et al, 2019 (Abrocitinib 200mg QD) & -55.82 & 32.83 & 38 & -35.91 & 47.71 & 7 & 1.8 & $-19.91[-56.76,16.94]$ & & 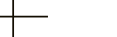 & \\
\hline Gooderham et al, 2019 (Abrocitinib 30mg QD) & -20.8 & 47.92 & 27 & -35.91 & 47.71 & 7 & 1.6 & $15.11[-24.59,54.81]$ & & & \\
\hline Guttman-Yassky et al, 2019 (Baricitinib 2mg QD) & -64.19 & 37.71 & 37 & -45.87 & 40.95 & 24 & 3.8 & $-18.32[-38.72,2.08]$ & & & \\
\hline Guttman-Yassky et al, 2019 (Baricitinib 4mg QD) & -64.68 & 38.28 & 38 & -45.87 & 40.95 & 25 & 3.8 & $-18.81[-38.95,1.33]$ & & & \\
\hline Guttman-Yassky et al, 2020 (Upadacitinib 15mg QD) & -61.7 & 39.66 & 42 & -23 & 40.09 & 13 & 3.1 & $-38.70[-63.58,-13.82]$ & & & \\
\hline Guttman-Yassky et al, 2020 (Upadacitinib 30mg QD) & -74.4 & 39.73 & 42 & -23 & 40.09 & 13 & 3.1 & $-51.40[-76.29,-26.51]$ & & & \\
\hline Guttman-Yassky et al, 2020 (Upadacitinib 7.5mg QD) & -39.4 & 40.44 & 42 & -23 & 40.09 & 13 & 3.0 & $-16.40[-41.39,8.59]$ & & & \\
\hline Kim et al, 2020 (Ruxolitinib 0.15\% QD) & -50.7 & 57.71 & 51 & -26.9 & 72.63 & 13 & 1.4 & $-23.80[-66.34,18.74]$ & & & \\
\hline Kim et al, 2020 (Ruxolitinib 0.5\% QD) & -58.5 & 55.93 & 51 & -26.9 & 72.63 & 13 & 1.4 & $-31.60[-73.96,10.76]$ & & - & \\
\hline Kim et al, 2020 (Ruxolitinib 1.5\% BID) & -78.5 & 28.71 & 50 & -26.9 & 72.63 & 13 & 1.6 & $-51.60[-91.88,-11.32]$ & & & \\
\hline Kim et al, 2020 (Ruxolitinib 1.5\% QD) & -67 & 39.46 & 52 & -26.9 & 72.63 & 13 & 1.5 & $-40.10[-81.01,0.81]$ & & & \\
\hline Nakagawa et al, 2018 (Delgocitinib 0.25\% BID) & -41.2 & 48.8 & 69 & -11.6 & 47 & 7 & 1.8 & $-29.60[-66.27,7.07]$ & & - & \\
\hline Nakagawa et al, 2018 (Delgocitinib 0.5\% BID) & -58.5 & 34.1 & 65 & -11.6 & 47 & 8 & 2.1 & $-46.90[-80.51,-13.29]$ & & & \\
\hline Nakagawa et al, 2018 (Delgocitinib 1\% BID) & -54.4 & 42 & 66 & -11.6 & 47 & 8 & 2.0 & $-42.80[-76.91,-8.69]$ & & & \\
\hline Nakagawa et al, 2018 (Delgocitinib 3\% BID) & -72.9 & 26.1 & 65 & -11.6 & 47 & 8 & 2.1 & $-61.30[-94.48,-28.12]$ & & & \\
\hline Nakagawa et al, 2019 (Delgocitinib 0.25\% BID) & -56 & 39.8 & 34 & -5.7 & 60.3 & 17 & 2.2 & $-50.30[-81.93,-18.67]$ & & & \\
\hline Nakagawa et al, 2019 (Delgocitinib 0.5\% BID) & -62.7 & 35 & 34 & -5.7 & 60.3 & 18 & 2.4 & $-57.00[-87.24,-26.76]$ & & & \\
\hline Nakagawa et al, 2020 (Delgocitinib 0.5\% BID) & -45.4 & 38.5 & 106 & -2.3 & 41 & 52 & 5.3 & $-43.10[-56.44,-29.76]$ & & & \\
\hline NCT03733301, 2020 (Baricitinib 2mg QD) & -58.16 & 36.89 & 100 & -45.08 & 36.32 & 45 & 5.4 & $-13.08[-25.92,-0.24]$ & & & \\
\hline NCT03733301, 2020 (Baricitinib 4mg QD) & -67.21 & 36.79 & 100 & -45.08 & 36.32 & 45 & 5.4 & $-22.13[-34.96,-9.30]$ & & & \\
\hline Silverberg et al, 2020 (Abrocitinib 100mg QD) & -60 & 41.37 & 158 & -28.6 & 44.16 & 39 & 4.8 & $-31.40[-46.69,-16.11]$ & & & \\
\hline Silverberg et al, 2020 (Abrocitinib 200mg QD) & -73.3 & 40.65 & 155 & -28.6 & 44.16 & 39 & 4.8 & $-44.70[-59.97,-29.43]$ & & & \\
\hline Simpson et al, BREEZE-AD1 2020 (Baricitinib 1mg QD) & -48.2 & 50.71 & 127 & -34.8 & 56.81 & 83 & 4.9 & $-13.40[-28.47,1.67]$ & & & \\
\hline Simpson et al, BREEZE-AD1 2020 (Baricitinib 2mg QD) & -51.9 & 47.69 & 123 & -34.8 & 56.81 & 83 & 4.9 & $-17.10[-31.95,-2.25]$ & & & \\
\hline Simpson et al, BREEZE-AD1 2020 (Baricitinib 4mg QD) & -59.4 & 42.49 & 125 & -34.8 & 56.81 & 83 & 5.1 & $-24.60[-38.91,-10.29]$ & & & \\
\hline Simpson et al, BREEZE-AD2 2020 (Baricitinib 1mg QD) & -41.7 & 59.26 & 125 & -28.9 & 67.17 & 81 & 4.3 & $-12.80[-30.74,5.14]$ & & & \\
\hline Simpson et al, BREEZE-AD2 2020 (Baricitinib 2mg QD) & -54.8 & 55.45 & 123 & -28.9 & 67.17 & 81 & 4.3 & $-25.90[-43.51,-8.29]$ & & & \\
\hline Simpson et al, BREEZE-AD2 2020 (Baricitinib 4mg QD) & -54.9 & 51.02 & 123 & -28.9 & 67.17 & 82 & 4.4 & $-26.00[-43.11,-8.89]$ & & & \\
\hline Total $(95 \% \mathrm{Cl})$ & & & 2,264 & & & 978 & 100.0 & $-28.82[-34.48,-23.16]$ & 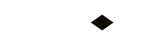 & & \\
\hline Heterogeneity: $\tau^{2}=111.53 ; \chi^{2}=59.78, \mathrm{df}=30(p=0.00$ & $0010) ; P^{2}=$ & $=50 \%$ & & & & & & 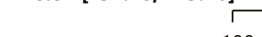 & 1 & 1 & $\longrightarrow$ \\
\hline Test for overall effect: $Z=9.98(p<0.00001)$ & & & & & & & & -100 & -50 & 50 & 100 \\
\hline & & & & & & & & & $\begin{array}{c}\text { Favours } \\
\text { JAK inhibitors }\end{array}$ & $\begin{array}{r}\text { Favo } \\
\text { Placebo/ }\end{array}$ & \\
\hline
\end{tabular}

Fig. 2. Meta-analysis for overall efficacy of treatment with Janus kinase (JAK) inhibitors in percent change from baseline on the Eczema Area and Severity Index versus placebo/vehicle.

olitinib, tofacitinib); among them, abrocitinib showed the most significant improvement in IGA response compared to placebo/vehicle (RR 5.47, 95\% CI 2.74$10.93, p<0.001)$. This result was confirmed at other measurement time points, with no heterogeneity (Table 2).

Regarding EASI scores, among the 6 JAK inhibitors assessed, namely, abrocitinib, baricitinib, delgocitinib, ruxolitinib, upadacitinib, and tofacitinib, upadacitinib provided the largest significant reduction compared to placebo/vehicle (WMD -53.92, 95\% CI -69.26 to -38.58 , $p<0.001)$. This result was confirmed at other measurement time points as well, and no significant heterogeneity $\left(I^{2}=31 \%\right)$ was detected (Table 3$)$.

\section{Safety Outcomes}

Adverse Effects Leading to Discontinuation

AEs were reported in 11 studies, which corresponded to 3,626 patients treated with $6 \mathrm{JAK}$ inhibitors, viz. abrocitinib, baricitinib, delgocitinib, ruxolitinib, tofacitinib, and upadacitinib. The most frequently reported AEs leading to discontinuation were eczema, $\mathrm{AD}$, headache, and abdominal pain (online supplementary Table $3 \mathrm{a}$ ). There were no significant differences in the number of AEs between the JAK inhibitors and placebo/vehicle (RR $0.79,95 \%$ CI $0.57-1.10, p=0.16$ ), with no significant heterogeneity $\left(I^{2}=34 \%\right.$; online supplementary Table $\left.4 \mathrm{a}\right)$.

Treatment-Emergent Adverse Effects

TEAEs were recorded in 11 studies that included 3,455 patients treated with 6 JAK inhibitors, i.e. abrocitinib, 
Table 2. Subgroup analysis of JAK inhibitors for treatment of atopic dermatitis in patients achieving Investigator's Global Assessment (IGA) response

\begin{tabular}{|c|c|c|c|c|c|}
\hline Subgroup & $\begin{array}{l}\text { Comparison, } \\
n\end{array}$ & $\begin{array}{l}\text { Treatment/control } \\
\text { participants, } n\end{array}$ & RR [95\% CI] & $p$ value & $I^{2}, \%$ \\
\hline \multicolumn{6}{|l|}{ At week 1} \\
\hline Total & 11 & $988 / 581$ & $2.36[1.05,5.32]$ & 0.04 & 0 \\
\hline Oral & 10 & $953 / 547$ & $1.59[0.65,3.90]$ & 0.32 & 0 \\
\hline Abrocitinib & 4 & $207 / 54$ & $0.96[0.21,4.51]$ & 0.96 & 0 \\
\hline Baricitinib & 6 & $746 / 493$ & $1.99[0.65,6.14]$ & 0.23 & 0 \\
\hline Topical & 1 & $35 / 34$ & $14.58[0.87,245.80]$ & 0.06 & NA \\
\hline Tofacitinib & 1 & $35 / 34$ & $14.58[0.87,245.80]$ & 0.06 & NA \\
\hline \multicolumn{6}{|l|}{ At week 2} \\
\hline Total & 19 & $1,809 / 787$ & $2.82[1.75,4.52]$ & $<0.001$ & 0 \\
\hline Oral & 14 & $1,570 / 701$ & $2.60[1.49,4.54]$ & $<0.001$ & 0 \\
\hline Abrocitinib & 8 & $824 / 208$ & $3.53[1.44,8.66]$ & 0.006 & 0 \\
\hline Baricitinib & 6 & $746 / 493$ & $1.99[0.98,4.03]$ & 0.06 & 0 \\
\hline Topical & 5 & $239 / 86$ & $3.53[1.43,8.73]$ & 0.006 & 0 \\
\hline Ruxolitinib & 4 & $204 / 52$ & $1.85[0.51,6.74]$ & 0.35 & 0 \\
\hline Tofacitinib & 1 & $35 / 34$ & $6.80[1.67,27.70]$ & 0.007 & NA \\
\hline \multicolumn{6}{|l|}{ At week 4} \\
\hline Total & 33 & $2,566 / 1,072$ & $3.59[2.66,4.84]$ & $<0.001$ & 0 \\
\hline Oral & 21 & $1,888 / 868$ & $3.65[2.53,5.27]$ & $<0.001$ & 0 \\
\hline Abrocitinib & 8 & $820 / 208$ & $5.47[2.74,10.93]$ & $<0.001$ & 0 \\
\hline ASN002 & 3 & $27 / 9$ & $1.61[0.34,7.69]$ & 0.55 & 0 \\
\hline Baricitinib & 10 & $1,041 / 651$ & $2.99[1.92,4.65]$ & $<0.001$ & 0 \\
\hline Topical & 12 & $678 / 204$ & $3.43[2.04,5.78]$ & $<0.001$ & 0 \\
\hline Delgocitinib & 7 & $439 / 118$ & $2.84[1.23,6.56]$ & 0.01 & 0 \\
\hline Ruxolitinib & 4 & $204 / 52$ & $2.68[1.01,7.15]$ & 0.05 & 0 \\
\hline Tofacitinib & 1 & $35 / 34$ & $5.83[2.26,15.03]$ & $<0.001$ & NA \\
\hline \multicolumn{6}{|l|}{ At week 8} \\
\hline Total & 14 & $1,104 / 309$ & $2.93[2.01,4.28]$ & $<0.001$ & 0 \\
\hline Oral & 10 & $900 / 257$ & $2.85[1.87,4.35]$ & $<0.001$ & 0 \\
\hline Abrocitinib & 8 & $825 / 208$ & $3.37[2.09,5.41]$ & $<0.001$ & 0 \\
\hline Baricitinib & 2 & $75 / 49$ & $0.98[0.37,2.58]$ & 0.97 & 0 \\
\hline Topical & 4 & $204 / 52$ & $3.27[1.40,7.67]$ & 0.006 & 0 \\
\hline Ruxolitinib & 4 & $204 / 52$ & $3.27[1.40,7.67]$ & 0.006 & 0 \\
\hline \multicolumn{6}{|l|}{ At week 12} \\
\hline Total & 10 & $887 / 254$ & $3.59[2.33,5.54]$ & $<0.001$ & 0 \\
\hline Oral & 10 & $887 / 254$ & $3.59[2.33,5.54]$ & $<0.001$ & 0 \\
\hline Abrocitinib & 8 & $812 / 205$ & $3.86[2.40,6.19]$ & $<0.001$ & 0 \\
\hline Baricitinib & 2 & $75 / 49$ & $2.13[0.74,6.14]$ & 0.16 & 0 \\
\hline \multicolumn{6}{|l|}{ At week 16} \\
\hline Total & 14 & $1,168 / 679$ & $2.22[1.64,3.00]$ & $<0.001$ & 0 \\
\hline Oral & 14 & $1,168 / 679$ & $2.22[1.64,3.00]$ & $<0.001$ & 0 \\
\hline Abrocitinib & 4 & $127 / 28$ & $1.33[0.46,3.83]$ & 0.6 & 0 \\
\hline Baricitinib & 10 & $1,041 / 651$ & $2.32[1.69,3.18]$ & $<0.001$ & 0 \\
\hline
\end{tabular}

NA, not applicable; RR, relative risk

baricitinib, delgocitinib, ruxolitinib, tofacitinib, and upadacitinib. The most frequently reported TEAEs were nasopharyngitis, headache, upper respiratory tract infection, and nausea (online supplementary Table $3 \mathrm{~b}$ ). A significant difference was found between JAK inhibitors and placebo/vehicle (RR 1.08, 95\% CI 1.01-1.17, $p=0.03$ ), with no significant heterogeneity $\left(I^{2}=37 \%\right)$. Additionally, abrocitinib was associated with a significantly higher rate of TEAEs than placebo/vehicle (RR 1.25, 95\% CI $1.10-1.42, p=0.001$ ) (online supplementary Table $4 \mathrm{~b}$ ). 
Table 3. Subgroup analysis of JAK inhibitors for treatment of atopic dermatitis in percent change from baseline in the eczema area and severity index (EASI)

\begin{tabular}{|c|c|c|c|c|c|}
\hline Subgroup & $\begin{array}{l}\text { Comparison, } \\
n\end{array}$ & $\begin{array}{l}\text { Treatment/ } \\
\text { Control participants, } n\end{array}$ & $\begin{array}{l}\text { WMD } \\
(95 \% \mathrm{CI})\end{array}$ & $p$ value & $I^{2}, \%$ \\
\hline \multicolumn{6}{|l|}{ At week 1} \\
\hline Total & 5 & $240 / 88$ & $-15.97[-31.09,-0.85]$ & 0.04 & 78 \\
\hline Oral & 4 & $205 / 54$ & $-10.23[-22.82,2.36]$ & 0.11 & 56 \\
\hline Abrocitinib & 4 & $205 / 54$ & $-10.23[-22.82,2.36]$ & 0.11 & 56 \\
\hline Topical & 1 & $35 / 34$ & $-36.80[-49.75,-23.85]$ & $<0.001$ & NA \\
\hline Tofacitinib & 1 & $35 / 34$ & $-36.80[-49.75,-23.85]$ & $<0.001$ & NA \\
\hline \multicolumn{6}{|l|}{ At week 2} \\
\hline Total & 14 & $876 / 259$ & $-32.09[-40.89,-23.29]$ & $<0.001$ & 67 \\
\hline Oral & 9 & $637 / 173$ & $-28.30[-39.92,-16.69]$ & $<0.001$ & 77 \\
\hline Abrocitinib & 6 & $511 / 133$ & $-21.69[-36.69,-6.69]$ & 0.005 & 82 \\
\hline Upadacitinib & 3 & $126 / 40$ & $-42.61[-54.47,-30.74]$ & $<0.001$ & 2 \\
\hline Topical & 5 & $239 / 86$ & $-41.67[-52.22,-31.11]$ & $<0.001$ & 0 \\
\hline Ruxolitinib & 4 & $204 / 52$ & $-39.95[-54.85,-25.04]$ & $<0.001$ & 0 \\
\hline Tofacitinib & 1 & $35 / 34$ & $-43.40[-58.36,-28.44]$ & $<0.001$ & NA \\
\hline \multicolumn{6}{|l|}{ At week 4} \\
\hline Total & 21 & $1,312 / 369$ & $-42.00[-48.64,-35.36]$ & $<0.001$ & 40 \\
\hline Oral & 9 & $635 / 168$ & $-37.66[-49.06,-26.27]$ & $<0.001$ & 70 \\
\hline Abrocitinib & 6 & $509 / 128$ & $-30.59[-43.54,-17.65]$ & $<0.001$ & 69 \\
\hline Upadacitinib & 3 & $126 / 40$ & $-53.92[-69.26,-38.58]$ & $<0.001$ & 31 \\
\hline Topical & 12 & $677 / 201$ & $-46.82[-54.56,-39.08]$ & $<0.001$ & 0 \\
\hline Delgocitinib & 7 & $439 / 118$ & $-45.98[-55.46,-36.49]$ & $<0.001$ & 0 \\
\hline Ruxolitinib & 4 & $204 / 52$ & $-44.06[-64.51,-23.61]$ & $<0.001$ & 0 \\
\hline Tofacitinib & 1 & $34 / 31$ & $-51.80[-69.50,-34.10]$ & $<0.001$ & NA \\
\hline \multicolumn{6}{|l|}{ At week 8} \\
\hline Total & 13 & $818 / 211$ & $-33.41[-43.20,-23.62]$ & $<0.001$ & 47 \\
\hline Oral & 9 & $614 / 159$ & $-32.33[-44.33,-20.34]$ & $<0.001$ & 63 \\
\hline Abrocitinib & 6 & $488 / 119$ & $-25.92[-39.32,-12.52]$ & $<0.001$ & 58 \\
\hline Upadacitinib & 3 & $126 / 40$ & $-46.66[-68.94,-24.38]$ & $<0.001$ & 61 \\
\hline Topical & 4 & $204 / 52$ & $-37.24[-57.98,-16.49]$ & $<0.001$ & 0 \\
\hline Ruxolitinib & 4 & $204 / 52$ & $-37.24[-57.98,-16.49]$ & $<0.001$ & 0 \\
\hline \multicolumn{6}{|l|}{ At week 12} \\
\hline Total & 9 & $632 / 169$ & $-33.30[-45.96,-20.63]$ & $<0.001$ & 63 \\
\hline Oral & 9 & $632 / 169$ & $-33.30[-45.96,-20.63]$ & $<0.001$ & 63 \\
\hline Abrocitinib & 6 & $506 / 130$ & $-26.95[-42.05,-11.85]$ & $<0.001$ & 63 \\
\hline Upadacitinib & 3 & $126 / 39$ & $-46.49[-68.64,-24.35]$ & $<0.001$ & 60 \\
\hline \multicolumn{6}{|l|}{ At week 16} \\
\hline Total & 17 & $1,274 / 699$ & $-19.82[-24.71,-14.94]$ & $<0.001$ & 9 \\
\hline Oral & 17 & $1,274 / 699$ & $-19.82[-24.71,-14.94]$ & $<0.001$ & 9 \\
\hline Abrocitinib & 4 & $127 / 28$ & $-2.20[-21.60,17.20]$ & 0.82 & 0 \\
\hline Baricitinib & 10 & $1,021 / 632$ & $-19.05[-24.01,-14.08]$ & $<0.001$ & 0 \\
\hline Upadacitinib & 3 & $126 / 39$ & $-35.53[-55.55,-15.50]$ & $<0.001$ & 48 \\
\hline
\end{tabular}

NA, not applicable; WMD, weighted mean difference

Publication Bias Assessment

Funnel plot analysis and Egger linear regression test demonstrated no evidence of publication bias for IGA response (bias $-0.14,95 \% \mathrm{CI}-0.80$ to $0.52, p=0.67$ ) and percent change from baseline in EASI (bias -0.53 , 95\% CI -1.90 to $0.83, p=0.43$ ) (online supplementary Fig. $3)$.

\section{Discussion}

To the best of our knowledge, this is the first metaanalysis to assess both the efficacy and safety of JAK inhibitors for treating AD. Our results show that, compared to placebo, JAK inhibitor treatment can significantly improve $\mathrm{AD}$ with acceptable safety. 
The use of JAK inhibitors to treat $\mathrm{AD}$ is gaining attention. A qualitative synthesis by Nusbaum et al. [39] demonstrated promising results in $\mathrm{AD}$ patients treated with oral or topical JAK inhibitors; however, that study lacked quantitative data analysis. A pooled meta-analysis of 4 RCTs [26, 31, 32, 34] by Arora et al. [40] included 534 patients and showed greater effectiveness of JAK inhibitors in $\mathrm{AD}$ treatment; however, the number of patients included was too small to draw strong conclusions. In our study, 10 RCTs [23-25, 27-30, 33, 35] including 3,288 patients were assessed for the first time, adding to the statistical power of the study to determine efficacy and safety. Given the wide spectrum of immunosuppressive effects of JAK inhibition [41], the safety of JAK inhibitor treatment was also assessed. Further, there was no detectable publication bias in the efficacy and safety of the outcomes. Our study confirms the effectiveness and safety of JAK inhibitors for AD treatment and is more believable.

There were some new findings from our subgroup analyses that had not been investigated in previous reports. First, a treatment duration analysis showed that JAK inhibitors exhibited significant effects as early as week 1 of treatment. The optimal duration was 4 weeks, implying that JAK inhibitors may be highly efficacious with rapid onset of action and clinically meaningful improvement. This rapid onset of action may be due to inhibition of JAK-cytokine receptor binding, which simultaneously blocks multiple cytokine signal transduction pathways $[42,43]$. Analysis of available data shows that the best effect of treatment with JAK inhibitors occurs at the fourth week. However, as most studies on topical JAK inhibitors had a treatment duration of 4 weeks, we could not confirm if this result will persist in longer treatment duration, and further trials with longer duration are needed to validate this result. Beyond 4 weeks, a decreasing trend in the clinical improvement of $\mathrm{AD}$ was observed in both IGA response and EASI score, except at week 12, which reached the same IGA response as in week 4. As $\mathrm{AD}$ has a chronic and relapsing disease course, additional studies are necessary to address whether JAK inhibitor treatment can be effective for longer than 16 weeks.

Second, we found that topical JAK inhibitors were associated with higher efficacy than oral therapy in improving both EASI score and IGA response at timepoints before week 12 except for week 4, where a discrepancy was found in improving IGA response. This discrepancy may be due to an inherent limitation in the IGA measurement tool, namely, lower interobserver reliability [44-46]. Nevertheless, as EASI has adequate validity and responsiveness $[47,48]$, our results on the greater efficacy of top-

JAK Inhibitors for the Treatment of Atopic Dermatitis ical JAK inhibitors remain credible. Next, subgroup analysis of the efficacy of topical versus oral administration, based on baseline severity of the treated subjects, showed that topical administration was more effective than oral administration in the moderate-to-severe subgroup in EASI. However, due to the paucity of data, efficacy was assessed only at week 4 in the moderate-to-severe group. Though oral administration displayed a better effect than topical administration with respect to IGA response, the number of participants in the oral administration group was much larger than the number of participants in the topical administration group; therefore, firm conclusions based on IGA response cannot be drawn.

Moreover, this observation has been confirmed in a murine model of allergic dermatitis, in which topical JAK inhibitors showed promising results against both pruritic and inflammatory responses; in contrast, oral JAK inhibitors could only decrease pruritus [49]. Nonetheless, it is noteworthy that the efficacy gap between oral and topical therapy gradually decreased over time. As data on the long-term efficacy of topical JAK inhibitors in AD treatment are not yet available, it remains unclear if topical administration will remain more effective than oral therapy after 12 weeks of treatment or later. Therefore, clinical trials on the long-term efficacy of topical JAK inhibitors in $\mathrm{AD}$ treatment are required.

This meta-analysis indicated that, based on the EASI score, upadacitinib for 4 weeks was the most effective treatment. For the outcome of IGA response, we preferred the result that abrocitinib for 4 weeks achieved the optimal efficacy. Although topical application of tofacitinib for 2 weeks was the most effective treatment in improving the IGA response at all measurement time points. However, this should be interpreted with caution because the number of patients included in this subgroup was relatively small. Further, the reason for the variance in the clinical performance of the involved JAK inhibitors remains unknown, but variations in affinity and selectivity profile among JAK inhibitors for JAK isoforms may contribute to the observed variation in clinical performance $[50,51]$. When two JAK isoforms are involved in signaling from a given cytokine, e.g. JAK1/JAK 3 binds to IL$2 \mathrm{R}$, selective inhibition of one cytokine significantly impacts that cytokine's function $[52,53]$. Thus, studies and clinical trials on $\mathrm{AD}$ treatment that compare different combinations of JAK inhibitors are needed to assess this phenomenon better.

Except for abrocitinib, with significantly more TEAEs than placebo, all other JAK inhibitors displayed a favorable safety profile during short-term treatment. Never- 
theless, JAKs play a role in immune function and hematopoietic systems, including thromboembolic risks [54], and additional data from long-term studies are needed to adequately assess such risks.

Our study has some limitations. First, all included studies were short-term RCTs; only 5 were on topical JAK inhibitor treatment and had small sample sizes. Second, no firm conclusions could be drawn based on baseline severity because of insufficient data and significant differences in participant numbers. Third, both IGA and EASI are physician-assessed instruments, which measure only clinical signs and do not address patient experience with pruritus and sleeplessness. A simple assessment of lesions does not represent a comprehensive evaluation of $\mathrm{AD}$ severity, and patient-assessable instruments with a healthrelated quality of life survey are also needed in future studies.

\section{Conclusion}

The results of this study support the efficacy and safe use of JAK inhibitors for AD treatment. Further trials with longer duration and head-to-head comparisons of different JAK inhibitors are needed to establish the most appropriate treatment for AD using JAK inhibitors.

\section{Key Message}

JAK inhibitors are effective and safe for AD treatment.

\section{Statement of Ethics}

This study was conducted in accordance with the Preferred Reporting Items for Systematic Reviews and Meta-analyses (PRIS$\mathrm{MA}$ ) and registered in PROSPERO with registration number CRD42020215945.

\section{Conflict of Interest Statement}

The authors have no conflicts of interest to disclose.

\section{Funding Sources}

No funding was received for this study.

\section{Author Contributions}

C.L. conceptualized the study, interpreted data, and drafted the initial manuscript; X.S. and K.Z. conceptualized ideas and edited the manuscript; F.M. and L.L. supervised the analysis; Z.M. helped in data extraction and analysis; X.H. conceptualized the study and reviewed the manuscript; all authors approved the final manuscript for submission.

\section{References}

1 Langan SM, Irvine AD, Weidinger S. Atopic dermatitis. Lancet. 2020 Aug;396(10247):345-60.

2 Barbarot S, Auziere S, Gadkari A, Girolomoni G, Puig L, Simpson EL, et al. Epidemiology of atopic dermatitis in adults: results from an international survey. Allergy. 2018 Jun;73(6):1284-93.

3 Nutten S. Atopic dermatitis: global epidemiology and risk factors. Ann Nutr Metab. 2015; 66(Suppl 1):8-16.

4 Schonmann Y, Mansfield KE, Hayes JF, Abuabara K, Roberts A, Smeeth L, et al. Atopic Eczema in Adulthood and Risk of Depression and Anxiety: A Population-Based Cohort Study. J Allergy Clin Immunol Pract. 2020 Jan;8(1):248-e16.

5 Patel KR, Immaneni S, Singam V, Rastogi S, Silverberg JI. Association between atopic dermatitis, depression, and suicidal ideation: A systematic review and meta-analysis. J Am Acad Dermatol. 2019 Feb;80(2):402-10.

6 Sandhu JK, Wu KK, Bui TL, Armstrong AW. Association Between Atopic Dermatitis and Suicidality: A Systematic Review and Meta-Analysis. JAMA Dermatol. 2019 Feb;155(2):178-87.

7 Eckert L, Gupta S, Amand C, Gadkari A, Mahajan P, Gelfand JM. Impact of atopic dermatitis on health-related quality of life and productivity in adults in the United States: An analysis using the National Health and Wellness Survey. J Am Acad Dermatol. 2017 Aug; 77(2):274-9.

8 Wollenberg A, Barbarot S, Bieber T, ChristenZaech S, Deleuran M, Fink-Wagner A, et al. Consensus-based European guidelines for treatment of atopic eczema (atopic dermatitis) in adults and children: part I. J Eur Acad Dermatol Venereol. 2018 May;32(5):657-82.

9 Sidbury R, Davis DM, Cohen DE, Cordoro KM, Berger TG, Bergman JN, et al. Guidelines of care for the management of atopic dermatitis: section 3. Management and treatment with phototherapy and systemic agents. J Am Acad Dermatol. 2014 Aug;71(2):327-49.

10 Moyle M, Cevikbas F, Harden JL, Guttman-Yassky E. Understanding the immune landscape in atopic dermatitis: The era of biologics and emerging therapeutic approaches. Exp Dermatol. 2019 Jul;28(7):756-68.

11 Strand V, Balsa A, Al-Saleh J, Barile-Fabris L, Horiuchi T, Takeuchi T, et al. Immunogenicity of Biologics in Chronic Inflammatory Diseases: A Systematic Review. BioDrugs. 2017 Aug;31(4): 299-316.

12 Chun PIF, Lehman H. Current and Future Monoclonal Antibodies in the Treatment of
Atopic Dermatitis. Clin Rev Allergy Immunol. 2020 Oct;59(2):208-19.

13 Kim J, Kim BE, Leung DYM. Pathophysiology of atopic dermatitis: Clinical implications. Allergy Asthma Proc. 2019 Mar;40(2):84-92.

14 Solimani F, Meier K, Ghoreschi K. Emerging Topical and Systemic JAK Inhibitors in Dermatology. Front Immunol. 2019 Dec;10:2847-66.

15 O'Shea JJ, Schwartz DM, Villarino AV, Gadina M, McInnes IB, Laurence A. The JAK-STAT pathway: impact on human disease and therapeutic intervention. Annu Rev Med. 2015 Jan; 66:311-28.

16 Oetjen LK, Mack MR, Feng J, Whelan TM, Niu $\mathrm{H}$, Guo CJ, et al. Sensory Neurons Co-opt Classical Immune Signaling Pathways to Mediate Chronic Itch. Cell. 2017 Sep;171(1):217-e13.

17 Amano W, Nakajima S, Kunugi H, Numata Y, Kitoh A, Egawa G, et al. The Janus kinase inhibitor JTE-052 improves skin barrier function through suppressing signal transducer and activator of transcription 3 signaling. J Allergy Clin Immunol. 2015 Sep;136(3):667-e7.

18 Phan K, Sebaratnam DF. JAK inhibitors for alopecia areata: a systematic review and meta-analysis. J Eur Acad Dermatol Venereol. 2019 May; 33(5):850-6. 
19 Kuo CM, Tung TH, Wang SH, Chi CC. Efficacy and safety of tofacitinib for moderate-tosevere plaque psoriasis: a systematic review and meta-analysis of randomized controlled trials. J Eur Acad Dermatol Venereol. 2018 Mar; 32(3):355-62.

20 Moher D, Liberati A, TetzlaffJ, Altman DG. Preferred reporting items for systematic reviews and meta-analyses: the PRISMA statement. BMJ. 2009 Jul;339:b2535.

21 Liberati A, Altman DG, Tetzlaff J, Mulrow C, Gøtzsche PC, Ioannidis JP, et al. The PRISMA statement for reporting systematic reviews and meta-analyses of studies that evaluate healthcare interventions: explanation and elaboration. BMJ. 2009 Jul;339:b2700.

22 Higgins JPT. TJ, Chandler J, Cumpston M, Li T, Page MJ, Welch VA (editors). Cochrane Handbook for Systematic Reviews of Interventions version 6.1 [cited $2021 \mathrm{Feb} 4$ ]. Available from: www.training.cochrane.org/handbook.

23 Gooderham MJ, Forman SB, Bissonnette R, Beebe JS, Zhang W, Banfield C, et al. Efficacy and Safety of Oral Janus Kinase 1 Inhibitor Abrocitinib for Patients With Atopic Dermatitis: A Phase 2 Randomized Clinical Trial. JAMA Dermatol. 2019 Oct;155(12):1371-9.

24 Silverberg JI, Simpson EL, Thyssen JP, Gooderham M, Chan G, Feeney C, et al. Efficacy and Safety of Abrocitinib in Patients With Moderateto-Severe Atopic Dermatitis: A Randomized Clinical Trial. JAMA Dermatol. 2020 Jun;156(8): 863-73.

25 Simpson EL, Sinclair R, Forman S, Wollenberg A, Aschoff R, Cork M, et al. Efficacy and safety of abrocitinib in adults and adolescents with moderate-to-severe atopic dermatitis (JADE MONO-1): a multicentre, double-blind, randomised, placebo-controlled, phase 3 trial. Lancet. 2020 Jul;396(10246):255-66.

26 Bissonnette R, Maari C, Forman S, Bhatia N, Lee M, Fowler J, et al. The oral Janus kinase/ spleen tyrosine kinase inhibitor ASN002 demonstrates efficacy and improves associated systemic inflammation in patients with moderate-to-severe atopic dermatitis: results from a randomized double-blind placebocontrolled study. Br J Dermatol. 2019 Oct; 181(4):733-42.

27 Guttman-Yassky E, Silverberg JI, Nemoto O, Forman SB, Wilke A, Prescilla R, et al. Baricitinib in adult patients with moderate-to-severe atopic dermatitis: A phase 2 parallel, double-blinded, randomized placebo-controlled multiple-dose study. J Am Acad Dermatol. 2019 Apr;80(4): 913-e9.

28 A Study of Baricitinib (LY3009104) in Combination With Topical Corticosteroids in Adults With Moderate to Severe Atopic Dermatitis (BREEZE-AD7) NCT03733301 [cited $2021 \mathrm{Feb}$ 4]. Available from: https://clinicaltrials.gov/ct2/ show/NCT03733301.
29 Simpson EL, Lacour JP, Spelman L, Galimberti R, Eichenfield LF, Bissonnette R, et al. Baricitinib in patients with moderate-to-severe atopic dermatitis and inadequate response to topical corticosteroids: results from two randomized monotherapy phase III trials. Br J Dermatol. 2020 Aug; 183(2):242-55.

30 Nakagawa $\mathrm{H}$, Nemoto O, Igarashi A, Saeki H, Kaino H, Nagata T. Delgocitinib ointment, a topical Janus kinase inhibitor, in adult patients with moderate to severe atopic dermatitis: A phase 3, randomized, double-blind, vehicle-controlled study and an open-label, long-term extension study. J Am Acad Dermatol. 2020 Apr; 82(4):823-31.

31 Nakagawa $\mathrm{H}$, Nemoto O, Igarashi A, Saeki H, Oda M, Kabashima K, et al. Phase 2 clinical study of delgocitinib ointment in pediatric patients with atopic dermatitis. J Allergy Clin Immunol. 2019 Dec;144(6):1575-83.

32 Nakagawa $\mathrm{H}$, Nemoto O, Igarashi $\mathrm{A}$, Nagata $\mathrm{T}$. Efficacy and safety of topical JTE-052, a Janus kinase inhibitor, in Japanese adult patients with moderate-to-severe atopic dermatitis: a phase II, multicentre, randomized, vehicle-controlled clinical study. Br J Dermatol. 2018 Feb;178(2): 424-32.

33 Kim BS, Howell MD, Sun K, Papp K, Nasir A, Kuligowski ME. Treatment of atopic dermatitis with ruxolitinib cream (JAK1/JAK2 inhibitor) or triamcinolone cream. J Allergy Clin Immunol. 2020 Feb;145(2):572-82.

34 Bissonnette R, Papp KA, Poulin Y, Gooderham M, Raman M, Mallbris L, et al. Topical tofacitinib for atopic dermatitis: a phase IIa randomized trial. Br J Dermatol. 2016 Nov;175(5):90211.

35 Guttman-Yassky E, Thaçi D, Pangan AL, Hong HC, Papp KA, Reich K, et al. Upadacitinib in adults with moderate to severe atopic dermatitis: 16-week results from a randomized, placebocontrolled trial. J Allergy Clin Immunol. 2020 Mar;145(3):877-84.

36 Hanifin JM, Rajka G. Diagnostic features of atopic dermatitis. Acta Derm Venereol. 1980(Suppl 92):44-7.

37 Eichenfield LF, Tom WL, Chamlin SL, Feldman SR, Hanifin JM, Simpson EL, et al. Guidelines of care for the management of atopic dermatitis: section 1. Diagnosis and assessment of atopic dermatitis. J Am Acad Dermatol. 2014 Feb; 70(2):338-51.

38 Saeki H, Nakahara T, Tanaka A, Kabashima K, Sugaya M, Murota $\mathrm{H}$, et al. Clinical Practice Guidelines for the Management of Atopic Dermatitis 2016. J Dermatol. 2016 Oct;43(10):111745.

39 Nusbaum KB, Nguyen CM, Fleischer AB Jr. Emerging systemic therapies for atopic dermatitis: oral small molecules and targeted topical agents. J Dermatolog Treat. 2020 Nov:1-5.

40 Arora CJ, Khattak FA, Yousafzai MT, Ibitoye BM, Shumack S. The effectiveness of Janus kinase inhibitors in treating atopic dermatitis: A systematic review and meta-analysis. Dermatol Ther. 2020 May;33(4):e13685.
41 Olivera PA, Lasa JS, Bonovas S, Danese S, Peyrin-Biroulet L. Safety of Janus Kinase Inhibitors in Patients With Inflammatory Bowel Diseases or Other Immune-mediated Diseases: A Systematic Review and Meta-Analysis. Gastroenterology. 2020 May;158(6):1554-e12.

42 Newsom M, Bashyam AM, Balogh EA, Feldman SR, Strowd LC. New and Emerging Systemic Treatments for Atopic Dermatitis. Drugs. 2020 Jul;80(11):1041-52.

43 Damsky W, King BA. JAK inhibitors in dermatology: The promise of a new drug class. J Am Acad Dermatol. 2017 Apr;76(4):736-44.

44 Bożek A, Reich A. Assessment of Intra- and Inter-Rater Reliability of Three Methods for Measuring Atopic Dermatitis Severity: EASI, Objective SCORAD, and IGA. Dermatology. 2017 May;233(1):16-22.

45 Chopra R, Silverberg JI. Assessing the severity of atopic dermatitis in clinical trials and practice. Clin Dermatol. 2018 Sep-Oct;36(5):606-15.

46 Futamura M, Leshem YA, Thomas KS, Nankervis $\mathrm{H}$, Williams $\mathrm{HC}$, Simpson EL. A systematic review of Investigator Global Assessment (IGA) in atopic dermatitis (AD) trials: Many options, no standards. J Am Acad Dermatol. 2016 Feb; 74(2):288-94

47 Schmitt J, Langan S, Deckert S, Svensson A, von Kobyletzki L, Thomas K, et al. Assessment of clinical signs of atopic dermatitis: a systematic review and recommendation. J Allergy Clin Immunol. 2013 Dec;132(6):1337-47.

48 Schmitt J, Spuls PI, Thomas KS, Simpson E, Furue $\mathrm{M}$, Deckert S, et al. The Harmonising Outcome Measures for Eczema (HOME) statement to assess clinical signs of atopic eczema in trials. J Allergy Clin Immunol. 2014 Oct;134(4):800-7.

49 Fukuyama T, Ehling S, Cook E, Bäumer W. Topically Administered Janus-Kinase Inhibitors Tofacitinib and Oclacitinib Display Impressive Antipruritic and Anti-Inflammatory Responses in a Model of Allergic Dermatitis. J Pharmacol Exp Ther. 2015 Sep;354(3):394-405.

50 Schwartz DM, Kanno Y, Villarino A, Ward M, Gadina M, O'Shea JJ. JAK inhibition as a therapeutic strategy for immune and inflammatory diseases. Nat Rev Drug Discov. 2017 Dec;17(12): 78-62.

51 Kettle JG, Åstrand A, Catley M, Grimster NP, Nilsson M, Su Q, et al. Inhibitors of JAK-family kinases: an update on the patent literature 2013 2015, part 1. Expert Opin Ther Pat. 2017 Feb; 27(2):127-43.

52 Moodley D, Yoshida H, Mostafavi S, Asinovski N, Ortiz-Lopez A, Symanowicz P, et al. Network pharmacology of JAK inhibitors. Proc Natl Acad Sci U S A. 2016 Aug;113(35):9852-7.

53 Garrido-Trigo A, Salas A. Molecular Structure and Function of Janus Kinases: Implications for the Development of Inhibitors. J Crohns Colitis. 2020 Aug;14(Suppliment_2):S713-s24.

54 Scott IC, Hider SL, Scott DL. Thromboembolism with Janus Kinase (JAK) Inhibitors for Rheumatoid Arthritis: How Real is the Risk? Drug Saf. 2018 Jul;41(7):645-53.
JAK Inhibitors for the Treatment of Atopic Dermatitis
Dermatology 2022;238:725-735

DOI: $10.1159 / 000518541$ 